\title{
Review of quarkonium production: status and prospects
}

\author{
Hee Sok Chung* \\ Physik-Department, Technische Universität München, James-Franck-Str. 1, 85748 Garching, \\ Germany \\ E-mail: heesok. chung@tum.de
}

\begin{abstract}
Production cross sections of heavy quarkonia are considered as useful tools to study various aspects of QCD. We have entered a new era of quarkonium production phenomenology, with the help of new measurements from the LHC giving access to more varieties of observables, and recent theoretical developments that provided us a better understanding of the short-distance process in which quarkonia are produced. With a more concrete understanding of perturbative corrections and a prolific collection of data, a satisfactory description of the quarkonium production mechanism might now be within reach. Unfortunately, the exact mechanism of quarkonium production still remains elusive. Even analyses based on the same formalism can lead to different descriptions of the production process and give contradicting predictions of processes involving heavy quarkonia. This implies that there are much more physics yet to be understood and much work yet to be done in quarkonium production phenomenology. In this paper, we review the current status of theoretical approaches and discuss possible strategies that may improve our understanding of heavy quarkonium production.
\end{abstract}

XIII Quark Confinement and the Hadron Spectrum - Confinement2018

31 July - 6 August 2018

Maynooth University, Ireland

${ }^{*}$ Speaker. 


\section{Introduction}

The many faces of QCD not only provide us a rich collection of phenomena to be understood but also a multitude of useful tools to study them. Hard processes producing heavy quarkonia, which are bound states of a heavy quark $Q$ and a heavy antiquark $\bar{Q}$, belong to both categories; while the hard scale lets us probe the perturbative side of $\mathrm{QCD}$, the formation of heavy quarkonium states involves physics at scales comparable or less than $\Lambda_{\mathrm{QCD}}$. Hence, heavy quarkonia are useful laboratories to study the interplay between perturbative and nonperturbative aspects of QCD [1,2]. Moreover, many heavy quarkonia have clean decay channels that enable precision measurements in colliders. Especially, the heavy quarkonium states with $J^{P C}=1^{--}$such as the $J / \psi, \psi(2 S)$, and the $\Upsilon(n S)$ can decay into lepton pairs. A good understanding of the heavy quarkonium production mechanism is also important in other areas of QCD, such as the study of the quark-gluon plasma, where the $J / \psi$ production cross sections in heavy ion collisions are used to probe the hot and dense phase of QCD [3]. The production mechanism of heavy quarkonia even has important phenomenological implications for beyond the standard model physics models with strongly interacting bound states [4].

A heavy quarkonium production process involves several distinguishable scales: there are the perturbative scales, which are the scale of the hard process and the scale of the heavy quark mass $m$, and there are the scales where QCD turns nonperturbative, which are the momentum $m v$ and the kinetic energy $m v^{2}$ of the heavy quark inside the heavy quarkonium. Here, $v$ is the typical velocity of the heavy quark inside the meson in the meson rest frame. For charmonium, $v^{2} \approx 0.3$, while for bottomonium, $v^{2} \approx 0.1$. When physical processes involve more than one scale that are strongly separated, the appropriate tool to study such processes are effective field theories. By integrating out the scales above $m v$, we obtain nonrelativistic QCD (NRQCD) [5, 6]. In NRQCD, the nonperturbative physics of scales $m v$ and $m v^{2}$ are contained in the NRQCD long-distance matrix elements (LDMEs), while the perturbative physics involving scales $m$ and above are captured in the corresponding short-distance coefficients (SDCs).

In NRQCD, the inclusive production cross section of a heavy quarkonium $H$ is given by the factorization formula [6]

$$
\sigma_{H}=\sum_{n} \sigma_{Q \bar{Q}(n)}\left\langle 0\left|\mathscr{O}^{H}(n)\right| 0\right\rangle,
$$

where $\sigma_{Q \bar{Q}(n)}$ is the SDC given by the inclusive production cross section of a perturbative $Q \bar{Q}$ state in a specific color and angular momentum state $n$, and $\left\langle 0\left|\mathscr{O}^{H}(n)\right| 0\right\rangle$ is the NRQCD LDME that governs the evolution of the $Q \bar{Q}$ in the color and angular momentum state $n$ into the heavy quarkonium $H$. The LDMEs have known scalings with $v$, and the sum over $n$ can be organized in powers of $v$. In practice, the sum is truncated at a desired accuracy in $v$. If NRQCD factorization holds in the form of Eq. (1.1), the LDMEs are universal, process-independent quantities that depend only on the properties of the quarkonium $H$. A theoretical prediction of a quarkonium production cross section requires perturbative calculations of the SDCs and nonperturbative determinations of the LDMEs. There have been great effort to make theoretical predictions for the production of $J / \psi$, partly due to the availability of experimental data over a wide range of kinematical configurations (see, for example, Ref. [2] and references therein). 
In present-day phenomenology of $J / \psi$ production, the factorization formula is usually truncated at relative order $v^{4}$. At leading order in $v$, the factorization formula involves the production of $c \bar{c}$ in the color-singlet, spin-triplet $S$-wave state $\left({ }^{3} S_{1}^{[1]}\right)$. Through order $v^{4}$, the factorization formula also involves the $c \bar{c}$ in the color-octet spin-singlet $S$-wave state $\left({ }^{1} S_{0}^{[8]}\right)$, in the color-octet spin-triplet $S$-wave state $\left({ }^{3} S_{1}^{[8]}\right)$, and in the color-octet spin-triplet $P$-wave states $\left({ }^{3} P_{J}^{[8]}\right)$ with $J=0,1,2$. Due to the approximate heavy-quark spin symmetry of NRQCD, the three color-octet $P$-wave LDMEs $\left\langle 0\left|\mathscr{O}^{J / \psi}\left({ }^{3} P_{J}^{[8]}\right)\right| 0\right\rangle$ can be given in terms of $\left\langle 0\left|\mathscr{O}^{J / \psi}\left({ }^{3} P_{0}^{[8]}\right)\right| 0\right\rangle$. Therefore, to relative order- $v^{4}$ accuracy, the production cross section of $J / \psi$ involves four NRQCD LDMEs and the corresponding SDCs. While the color-singlet LDME $\left\langle 0\left|\mathscr{O}^{J / \psi}\left({ }^{3} S_{1}^{[1]}\right)\right| 0\right\rangle$ can be obtained from decay rates of $J / \psi$, computed from potential models, or measured using lattice QCD, it is not yet known how to compute the color-octet LDMEs from first principles. Hence, the color-octet LDMEs are usually determined by comparing Eq. (1.1) with data.

Inclusive production cross section of $J / \psi$ have been measured in many experiments. For phenomenological studies, single $J / \psi$ inclusive cross sections as functions of the transverse momentum $p_{T}$ are preferred because a positive definite $p_{T}$ gives rise to a natural choice of the hard scale as $p_{T}$ or $m_{T}=\sqrt{p_{T}^{2}+m_{J / \psi}^{2}}$, where $m_{J / \psi}$ is the $J / \psi$ mass. The measured $p_{T}$-differential cross sections include the processes $e^{+} e^{-} \rightarrow J / \psi+X$ by the Belle experiment at KEKB [7], $e^{+} e^{-} \rightarrow e^{+} e^{-}+J / \psi+X$ (two-photon scattering) by DELPHI at LEP II [8], $e p \rightarrow J / \psi+X$ (photoproduction) by $\mathrm{H} 1$ and Zeus at HERA [9, 10, 11], and the hadroproduction $p \bar{p} \rightarrow J / \psi+X$ by CDF at Tevatron [12, 13], $p p \rightarrow J / \psi+X$ by PHENIX and STAR at RHIC [14, 15], and by CMS [16, 17, 18, 19], ATLAS [20, 21], ALICE [22, 23, 24, 25], and LHCb [26, 27, 28] experiments at the LHC. The SDCs for these processes have been computed to next-to-leading order (NLO) in $\alpha_{s}[29,30,31,32,33,34,35,36,37]$. Currently, there are a number of determinations of $J / \psi$ LDMEs available in literature that are based on the NLO SDCs. Among them, we introduce and compare four representative examples from Refs. [34, 35, 38, 39] that are based on the cross section measurements in Sec. 2. In Sec. 3, we compare the measurements and predictions of other observables that can serve as tests of the LDME determinations. We summarize in Sec. 4.

\section{Determination of NRQCD LDMEs from cross section data}

In this section, we present four representative examples of NRQCD LDME determinations from Refs. [34, 35, 38, 39], which are based on the SDCs at NLO accuracy. In these determinations, the color-octet LDMEs have been obtained by comparing the NRQCD factorization formula (1.1) with cross section measurements, while the color-singlet LDME have been taken from other determinations. Also, in these determinations, the feed-down contributions to the $J / \psi$ cross section from decays of $\psi(2 S), \chi_{c 1}$ and $\chi_{c 2}$ have been taken into account. In Refs. [35, 39], the colorsinglet LDME was taken to be the value $\left\langle 0\left|\mathscr{O}^{J / \psi}\left({ }^{3} S_{1}^{[1]}\right)\right| 0\right\rangle=1.32 \mathrm{GeV}^{3}$ which was determined in Ref. [40], and in Refs. [34, 38], the value $\left\langle 0\left|\mathscr{O}^{J / \psi}\left({ }^{3} S_{1}^{[1]}\right)\right| 0\right\rangle=1.16 \mathrm{GeV}^{3}$ from Ref. [41] was used. The difference between the two values is insignificant compared to the uncertainties in the SDC for the color-singlet channel, and hence, it is fair to say that the employed values of the color-singlet LDME are consistent with each other. Meanwhile, there are considerable differences in the coloroctet LDMEs. We show the color-octet LDMEs from Refs. [34, 35, 39] in Table 1, and present the color-octet LDMEs from Ref. [38] below. 


\begin{tabular}{c|ccc} 
& $\left\langle 0\left|\mathscr{O}^{J / \psi}\left({ }^{1} S_{0}^{[8]}\right)\right| 0\right\rangle\left(\mathrm{GeV}^{3}\right)$ & $\left\langle 0\left|\mathscr{O}^{J / \psi}\left({ }^{3} S_{1}^{[8]}\right)\right| 0\right\rangle\left(\mathrm{GeV}^{3}\right)$ & $\left\langle 0\left|\mathscr{O}^{J / \psi}\left({ }^{3} P_{0}^{[8]}\right)\right| 0\right\rangle\left(\mathrm{GeV}^{5}\right)$ \\
\hline Ref. [34] & $(9.7 \pm 0.9) \times 10^{-2}$ & $(-0.46 \pm 0.13) \times 10^{-2}$ & $(-2.1 \pm 0.6) \times 10^{-2}$ \\
\hline Ref. [35] & $(3.04 \pm 0.35) \times 10^{-2}$ & $(1.68 \pm 0.46) \times 10^{-3}$ & $(-9.08 \pm 1.61) \times 10^{-3}$ \\
\hline Ref. [39] & $(1.10 \pm 0.14) \times 10^{-1}$ & $(-7.13 \pm 3.64) \times 10^{-3}$ & $(-7.03 \pm 3.40) \times 10^{-3}$
\end{tabular}

Table 1: Various determinations of color-octet NRQCD LDMEs for $J / \psi$.

In Ref. [38], only certain linear combinations of the color-octet LDMEs could be determined, and the authors of Ref. [38] determined ranges of the LDMEs by assuming that all 3 color-octet LDMEs are positive. This leads to $0<\left\langle 0\left|\mathscr{O}^{J / \psi}\left({ }^{1} S_{0}^{[8]}\right)\right| 0\right\rangle<(7.4 \pm 1.9) \times 10^{-2} \mathrm{GeV}^{3},(0.05 \pm$ $0.02) \times 10^{-2} \mathrm{GeV}^{3}<\left\langle 0\left|\mathscr{O}^{J / \psi}\left({ }^{3} S_{1}^{[8]}\right)\right| 0\right\rangle<(1.11 \pm 0.27) \times 10^{-2} \mathrm{GeV}^{3}$, and $0<\left\langle 0\left|\mathscr{O}^{J / \psi}\left({ }^{3} P_{0}^{[8]}\right)\right| 0\right\rangle<$ $(4.27 \pm 1.10) \times 10^{-2} \mathrm{GeV}^{5}$. The values of these LDMEs are correlated in a way that when $\left\langle 0\left|\mathscr{O}^{J / \psi}\left({ }^{3} P_{0}^{[8]}\right)\right| 0\right\rangle$ reaches its maximum value, $\left\langle 0\left|\mathscr{O}^{J / \psi}\left({ }^{3} S_{1}^{[8]}\right)\right| 0\right\rangle$ is also maximized, while the LDME $\left\langle 0\left|\mathscr{O}^{J / \psi}\left({ }^{1} S_{0}^{[8]}\right)\right| 0\right\rangle$ becomes zero. In the opposite limit where $\left\langle 0\left|\mathscr{O}^{J / \psi}\left({ }^{3} P_{0}^{[8]}\right)\right| 0\right\rangle$ vanishes, $\left\langle 0\left|\mathscr{O}^{J / \psi}\left({ }^{1} S_{0}^{[8]}\right)\right| 0\right\rangle$ reaches its maximum, while $\left\langle 0\left|\mathscr{O}^{J / \psi}\left({ }^{3} S_{1}^{[8]}\right)\right| 0\right\rangle$ is minimized. Note that the assumption in Ref. [38] that all LDMEs are positive is in tension with the determinations in Refs. [34, 35, 39], where one or more of the color-octet LDMEs are negative.

The determination in Ref. [35] is a global fit of a number of measurements, including hadroproduction from PHENIX [14] at RHIC, CDF at Tevatron I [12] and Tevatron II [13], CMS [16], ATLAS [20], ALICE [22], and LHCb [26] at the LHC, photoproduction from ZEUS [9] and H1 [10] at HERA I and H1 [11] at HERA II, the two-photon scattering from DELPHI [8] at LEP II, and $e^{+} e^{-}$ annihilation from Belle [7] at KEKB. The authors of Ref. [35] excluded data with $p_{T}<1 \mathrm{GeV}$ for photoproduction and two-photon scattering, and data with $p_{T}<3 \mathrm{GeV}$ for hadroproduction since the authors determined that the perturbative calculations of the SDCs are not reliable in those kinematical ranges. With the exception of hadroproduction, for most of the measurements considered in Ref. [35], the $p_{T}$ of $J / \psi$ does not exceed $10 \mathrm{GeV}$.

On the other hand, the LDME determinations in Refs. [34, 38, 39] are based on the hadroproduction data only. The LDME determination in Ref. [34] is based on the CDF [13] and LHCb [26] cross section measurements. In Ref. [38], the $J / \psi$ LDMEs were estimated from the CDF data [13]. The extraction in Ref. [39] used CDF [13] and CMS [17, 18] data. The authors of Ref. [39] included, in addition to the SDCs at NLO accuracy, the leading-power (LP) fragmentation corrections including the leading logarithms of $p_{T} / m_{c}$ resummed to all orders in $\alpha_{S}$ that have significant impact on the shape of the SDCs in $p_{T}$. Here, $m_{c}$ is the mass of the charm quark. In Refs. [34, 38], the data with $p_{T}<7 \mathrm{GeV}$ was not considered in fit, whereas in Ref. [39], only data with $p_{T}>10 \mathrm{GeV}$ was used. These $p_{T}$ cuts are considerably higher than what was employed in Ref. [35], and removes most of the photoproduction data and data from lepton colliders from consideration. It has been found that the LDMEs extracted from high- $p_{T}$ hadroproduction data are in conflict with the H1 photoproduction data and the Belle data $[42,43]$.

The hadroproduction data employed in Refs. [34, 38, 39] are over wide kinematical ranges, where the $p_{T}$ of $J / \psi$ can even exceed $100 \mathrm{GeV}$. Since the $p_{T}$ cuts employed in Refs. [34, 38, 39] are larger than $m_{J / \psi}$, we can understand the shape of the $p_{T}$-differential cross section from its expansion in powers of $1 / p_{T}$. The LP contribution, which scales like $d \sigma^{\mathrm{LP}} / d p_{T}^{2} \sim 1 / p_{T}^{4}$, is given 
by the LP fragmentation, where a single energetic parton produced in a hard process evolves into a quarkonium [44]. The next-to-leading power (NLP) contribution, which scales like $d \sigma^{\mathrm{NLP}} / d p_{T}^{2} \sim$ $1 / p_{T}^{6}$, is given by NLP fragmentation, where a pair of energetic partons produced in a hard process evolves into a quarkonium [45, 46, 47]. The LP (NLP) fragmentation contribution is given by the convolution of the hard part and the single(double)-parton fragmentation function. The SDCs can also be approximated by linear combinations of the LP and the NLP fragmentation contributions, where the fragmentation functions govern the evolution of the partons into the $Q \bar{Q}$ of specific color and angular momentum states [48]. In the ${ }^{1} S_{0}^{[8]}$ and ${ }^{3} P_{0}^{[8]}$ channels, the SDCs at leading order in $\alpha_{s}$ do not contain LP fragmentation contributions, and in those channels, the LP fragmentation contributions first appear at NLO in $\alpha_{s}$ [49]. Hence, the NLO correction to the SDCs are enhanced by $p_{T}^{2} / m_{c}^{2}$, and the NLO $K$ factors are large and depend strongly on $p_{T}$. However, since the NLO SDCs already contain LP fragmentation contributions, corrections of higher orders in $\alpha_{s}$ can no longer receive enhancements from powers of $p_{T} / m_{c}$.

It is well known that at large $p_{T}$, the contribution from the ${ }^{3} S_{1}^{[1]}$ channel severely underestimates the hadroproduction data [50]. This can also be understood from the expansion in powers of $1 / p_{T}$. The ${ }^{3} S_{1}^{[1]}$ channel does not have a LP contribution until NLO in $\alpha_{s}$. However, even at NLO, the LP fragmentation contribution only involves the fragmentation of a charm quark. In the hard process, the production rate of an energetic charm quark is suppressed compared to the production rate of a gluon or a light quark. Hence, it is not until NNLO in $\alpha_{s}$ that the ${ }^{3} S_{1}^{[1]}$ channel receives a sizable contribution from LP fragmentation. On the other hand the color-octet channels receive LP fragmentation contributions from LO $\left({ }^{3} S_{1}^{[8]}\right.$ channel $)$ and NLO $\left({ }^{1} S_{0}^{[8]}\right.$ and ${ }^{3} P_{0}^{[8]}$ channels), and when compared to the color-singlet channel, the suppression from powers of $v$ can be overcome by enhancement from inverse powers of $\alpha_{s}$ at the scale of $p_{T}$. In all LDME determinations in Refs. [34, 35, 38, 39], the contributions from the color-octet channels dominate the hadroproduction rate.

The color-octet LDMEs in Refs. [34, 39] have uncertainties that are strongly correlated. In Ref. [38], only two linear combinations of the three color-octet LDMEs could be determined, and the ranges of the LDMEs were constrained by requiring the positivity of all three color-octet LDMEs. These imply that hadroproduction data cannot constrain all three color-octet LDMEs strongly. The origin of this problem is the fact that the three SDCs corresponding to the three coloroctet LDMEs are approximately linearly dependent [31]. Since each SDC can also be approximated by a linear combination of the LP and the NLP fragmentation contributions, there is always an approximate linear dependence between three SDCs at large $p_{T}$. In the next section, we will discuss the efforts to further constrain the ranges of the matrix elements from Ref. [38] by using other measurements as constraints.

It is worth noting that, in the LDME extractions in Refs. [34, 38, 39], the color-octet LDMEs $\left\langle 0\left|\mathscr{O}^{J / \psi}\left({ }^{3} S_{1}^{[8]}\right)\right| 0\right\rangle$ and $\left\langle 0\left|\mathscr{O}^{J / \psi}\left({ }^{3} P_{0}^{[8]}\right)\right| 0\right\rangle$ have same signs. The SDCs for the ${ }^{3} S_{1}^{[8]}$ and ${ }^{3} P_{0}^{[8]}$ channels have large leading-power contributions compared to the next-to-leading power contributions, and the shapes of the SDCs in $p_{T}$ are incompatible with hadroproduction data. Therefore it is necessary that the either the sum of contributions from the two channels are small, or there should be large cancellations between the contributions in order to describe the large- $p_{T}$ data. Since the SDCs for the ${ }^{3} S_{1}^{[8]}$ and ${ }^{3} P_{0}^{[8]}$ channels have opposite signs at large $p_{T}$, for cancellations to occur, the corresponding LDMEs need to have same signs as in Refs. [34, 38, 39]. On the other hand, 
the color-octet LDMEs $\left\langle 0\left|\mathscr{O}^{J / \psi}\left({ }^{3} S_{1}^{[8]}\right)\right| 0\right\rangle$ and $\left\langle 0\left|\mathscr{O}^{J / \psi}\left({ }^{3} P_{0}^{[8]}\right)\right| 0\right\rangle$ have opposite signs in Ref. [35], and so, the contributions from those channels add at large $p_{T}$. These qualitative differences in the color-octet LDMEs have interesting implications in the prediction of other observables. For example, for hadroproduction and photoproduction at $p_{T}$ comparable to $m_{J / \psi}$, the SDCs for the ${ }^{3} S_{1}^{[8]}$ and ${ }^{3} P_{0}^{[8]}$ channels are both positive. Hence, positive color-octet LDMEs $\left\langle 0\left|\mathscr{O}^{J / \psi}\left({ }^{3} S_{1}^{[8]}\right)\right| 0\right\rangle$ and $\left\langle 0\left|\mathscr{O}^{J / \psi}\left({ }^{3} P_{0}^{[8]}\right)\right| 0\right\rangle$ can enhance the cross section when $p_{T}$ is comparable to $m_{J / \psi}$, and lead to overestimation of the cross section [42]. Comparison between the predictions of LDME determinations for other observables such as the polarization of $J / \psi$ produced in hadron colliders will be discussed in the next section.

\section{Comparison with other observables}

The large number of $J / \psi$ produced in hadron colliders give us access to observables other than the production rate. These observables can be sensitive to the color-octet LDMEs in a different combination than the cross sections, and can provide independent tests of the color-octet LDMEs. In this section we consider three such examples that have been measured at the LHC : the polarization of $J / \psi$, the momentum distribution of $J / \psi$ inside a jet, and the production rate of $\eta_{c}$.

\subsection{Polarization of $J / \psi$}

The polarization of $J / \psi$ can be measured from the angular distribution of the muon pairs from the decay of $J / \psi$ in the meson rest frame. The axis that defines the polarization of $J / \psi$ is usually chosen to be the direction of the boost from the lab frame to the rest frame of the $J / \psi$ : such choice is called the center-of-mass helicity frame. The polarization of $J / \psi$ have been measured by CDF [51, 52] at Tevatron, and by ALICE [53, 54], CMS [55], and LHCb [56, 57] at the LHC. While the CDF Run I [51] and Run II [52] measurements are incompatible with each other, the measurements at the LHC are in reasonable agreement and imply that the $J / \psi$ 's produced in $p p$ collisions are almost unpolarized.

The polarization of $J / \psi$ is sensitive to the color-octet LDMEs. At large $p_{T}$, the $J / \psi$ produced in the ${ }^{3} S_{1}^{[8]}$ and ${ }^{3} P_{0}^{[8]}$ channels have strong transverse polarization, while the ${ }^{1} S_{0}^{[8]}$ channel produces unpolarized $J / \psi$, because the ${ }^{1} S_{0}^{[8]}$ state is isotropic. Hence, in order to produce unpolarized $J / \psi$, either the ${ }^{1} S_{0}^{[8]}$ channel should dominate the cross section, or the contributions form ${ }^{3} S_{1}^{[8]}$ and ${ }^{3} P_{0}^{[8]}$ channels must have large cancellations so that the production rate of transversely polarized $J / \psi$ is reduced. Hence, the color-octet LDMEs determined in Refs. [34, 38, 39], which feature large cancellations between the contributions from the ${ }^{3} S_{1}^{[8]}$ and ${ }^{3} P_{0}^{[8]}$ channels in the cross section, lead to predictions of small or almost vanishing $J / \psi$ polarization at large $p_{T}$. In contrast, the color-octet LDMEs determined in Ref. [35] lead to a prediction of transversely polarized $J / \psi$ at large $p_{T}$, because in Ref. [35], the contributions from ${ }^{3} S_{1}^{[8]}$ and ${ }^{3} P_{0}^{[8]}$ channels add and enhance the transverse cross section at large $p_{T}[33,42,58]$. This prediction does not agree with measurements at the LHC [55].

In Ref. [32], the authors presented a set of color-octet LDMEs that were constrained by fitting the high- $p_{T}$ CDF cross section and polarization data simultaneously. This simultaneous fit leads to a large $\left\langle 0\left|\mathscr{O}^{J / \psi}\left({ }^{1} S_{0}^{[8]}\right)\right| 0\right\rangle$, while $\left\langle 0\left|\mathscr{O}^{J / \psi}\left({ }^{3} S_{1}^{[8]}\right)\right| 0\right\rangle$ and $\left\langle 0\left|\mathscr{O}^{J / \psi}\left({ }^{3} P_{0}^{[8]}\right)\right| 0\right\rangle$ are small and positive. 
This result is compatible with the ranges of color-octet LDMEs obtained by the same authors in Ref. [38], while having much narrower ranges, and therefore the LDMEs in Ref. [32] can lead to more concrete predictions of observables. These LDMEs predict slightly longitudinal $J / \psi$ at the LHC, which is in tension with Ref. [38], which predicts slightly transverse $J / \psi$ at the LHC.

\subsection{Momentum distribution of $J / \psi$ in jet}

Another useful observable is the momentum distribution of a $J / \psi$ in a jet [59]. The momentum distribution can be measured as a function of $z$, which is the fraction of the momentum of $J / \psi$ compared to the momentum of the jet. The shape of this distribution is sensitive to the color-octet LDMEs; the ${ }^{3} S_{1}^{[8]}$ and ${ }^{3} P_{0}^{[8]}$ channels have distributions that rise as $z \rightarrow 1$, while in the ${ }^{1} S_{0}^{[8]}$ channel, the distribution falls as $z \rightarrow 1$. So far, the LHCb [60] and CMS [61] have measured the momentum distribution of $J / \psi$ in jet. Both measurements have a common feature that the distribution falls as $z \rightarrow 1$. Since ${ }^{3} S_{1}^{[8]}$ and ${ }^{3} P_{0}^{[8]}$ channels have rising distributions as $z \rightarrow 1$, in order to have a falling distribution as $z \rightarrow 1$ we need a large cancellation between the ${ }^{3} S_{1}^{[8]}$ and ${ }^{3} P_{0}^{[8]}$ channels, or the ${ }^{1} S_{0}^{[8]}$ channel must dominate the cross section. In Ref. [62], the authors presented a calculation of the momentum distribution of $J / \psi$ in jet based on the fragmenting jet functions (FJF) formalism developed in Ref. [63]. The results from the FJF formalism are found to agree with calculations based on PYTHIA with modifications to properly accommodate the gluon fragmentation process [62, 64]. The authors of Ref. [62] made predictions based on three independent determinations of color-octet LDMEs based on the NLO calculation of the SDCs : LDME set 1 is from Refs. [35, 42], which is determined from the global fit of $J / \psi$ inclusive cross sections without considering feed-down contributions. LDME set 2 is from Ref. [32], where the LDMEs were obtained by fitting the high- $p_{T}$ CDF cross section and polarization data simultaneously. LDME set 2 is compatible with Ref. [38]. Finally, LDME set 3 from Ref. [49], where the LDMEs were obtained from high- $p_{T}$ cross section data from CDF and CMS, were also considered. The LDMEs in set 3 are compatible with those in Ref. [39]. Recall that, in Refs. [31, 38, 39], there are large cancellations between the ${ }^{3} S_{1}^{[8]}$ and ${ }^{3} P_{0}^{[8]}$ channels, and in Ref. [39], the ${ }^{1} S_{0}^{[8]}$ channel dominates the hadroproduction cross section. The same holds true for LDME sets 2 and 3, so that the resulting distributions fall as $z \rightarrow 1$; the overall shapes of the distributions are in reasonable agreement with data [62]. On the other hand, it was shown in Ref. [62] that the LDME set 1 gives a much flatter distribution that disagrees with data. It has not been reported whether the full ranges of color-octet LDMEs from Ref. [38] lead to a concrete prediction of the $J / \psi$ momentum distribution in jet.

\subsection{Production rate of $\eta_{c}$}

Due to the approximate heavy-quark spin symmetry of NRQCD, the LDMEs for the production of $J / \psi$ can be related with the LDMEs for the production of $\eta_{c}$ [6]. Hence, a determination of $J / \psi$ LDMEs leads to prediction of the $\eta_{c}$ production rate. The $p_{T}$-differential cross section of $\eta_{c}$ has been measured by LHCb [65], and so, this measurement can serve as a test of the $J / \psi$ production mechanism.

The $\eta_{c}$ production cross section involves at leading order in $v$ the color-singlet spin-singlet channel $\left({ }^{1} S_{0}^{[1]}\right)$, and through relative order $v^{4}$ the three color-octet channels ${ }^{3} S_{1}^{[8]},{ }^{1} P_{1}^{[8]}$, and ${ }^{1} S_{0}^{[8]}$. The corresponding LDMEs can be obtained from $J / \psi$ LDMEs that differ by one unit of spin, with 
appropriate conversion factors obtained from spin multiplicities of the LDMEs. On the other hand, the behavior of the SDCs are different from the case of $J / \psi$ hadroproduction; for $\eta_{c}$ hadroproduction at large $p_{T}$, the SDC for the color-singlet channel does not have the same strong suppression from $\alpha_{s}$ as the color-singlet SDC for the $J / \psi$, and even the contribution from the color-singlet channel is large enough to fully accommodate the $\eta_{c}$ hadroproduction data [66]. Moreover, the SDCs for the color-octet channels are positive for all channels, and so, the color-octet LDMEs that lead to large cancellations in the $J / \psi$ production rate can give large enhancements to the $\eta_{c}$ production rate. Also, since the SDC for the ${ }^{3} S_{1}^{[8]}$ channel is much larger than the SDC for the ${ }^{1} S_{0}^{[8]}$ channel, if the LDME $\left\langle 0\left|\mathscr{O}^{J / \psi}\left({ }^{1} S_{0}^{[8]}\right)\right| 0\right\rangle \approx\left\langle 0\left|\mathscr{O}^{\eta_{c}}\left({ }^{3} S_{1}^{[8]}\right)\right| 0\right\rangle$ is large, the contribution from the ${ }^{3} S_{1}^{[8]}$ channel to the $\eta_{c}$ cross section can be much larger than the contribution from the ${ }^{1} S_{0}^{[8]}$ channel to the $J / \psi$ cross section. Hence, if the $J / \psi$ production cross section is dominated by the ${ }^{1} S_{0}^{[8]}$ channel, the $\eta_{c}$ cross section will be strongly enhanced by the ${ }^{3} S_{1}^{[8]}$ channel.

The authors of Ref. [66] found that, the LDMEs from the global fit of $J / \psi$ cross sections [35], the LDMEs from simultaneous fit to production rate and polarization of $J / \psi$ at the Tevatron [32], and the LDMEs from $J / \psi$ hadroproduction data $[34,49]$ lead to predictions that overestimate the $\eta_{c}$ cross section data. The LDMEs from Ref. [38], which were obtained from the CDF $J / \psi$ cross section data with the positivity assumption, is found to be compatible with data $[67,68]$. In Refs. [67, 68], it has been shown to be possible to reduce the ranges of the color-octet LDMEs in Ref. [38] by using the $\eta_{c}$ cross section data. However, the LDMEs determined in Refs. [67, 68] have much smaller values of $\left\langle 0\left|\mathscr{O}^{J / \psi}\left({ }^{1} S_{0}^{[8]}\right)\right| 0\right\rangle$, and are in conflict with the LDMEs from Ref. [32] determined from the CDF cross section and polarization data. Also, the authors of Ref. [66] have pointed out that the prediction for the $J / \psi$ polarization based on the LDMEs in Ref. [67] is in tension with the LHC data.

\section{Summary and outlook}

In this paper we have reviewed the current status of $J / \psi$ production phenomenology based on the NRQCD factorization formalism. A prediction based on the NRQCD factorization formalism requires knowledge of the short-distance coefficients, which are perturbatively calculable, and the long-distance matrix elements, which are nonperturbative quantities. Since it is not known how to compute color-octet long-distance matrix elements, they are usually determined phenomenologically by comparing the NRQCD factorization formula with measurements. We presented four representative examples of the long-distance matrix element determinations from cross section measurements, based on the state-of-the-art calculations of the short-distance coefficients at nextto-leading order accuracy. The long-distance matrix elements determined from different choices of data can disagree with one another, and none of the determinations are able to give a comprehensive description of the important observables, such as the cross section measurements at various kinematical configurations, polarization, momentum distribution of $J / \psi$ in jet, and the $\eta_{c}$ cross section data at the LHC, at a satisfactory level. It is therefore unclear whether a prediction of an observable based a specific determination of the long-distance matrix elements can be regarded trustworthy, except for cases where the observable is insensitive to specific values of the coloroctet long-distance matrix elements at the current level of experimental accuracy (see, for example, Ref. [69]). 
One may question the reliability of the perturbative expansion of the short-distance coefficients. At small $p_{T}, \alpha_{s}$ becomes large, while at $p_{T}$ much larger than $m_{c}$, large corrections enhanced by powers and logarithms of $p_{T} / m_{c}$ may appear. In both cases, the convergence of the perturbation series can be spoiled. However, the next-to-leading order corrections to the short-distance coefficients show that, when $p_{T}$ is of the order of the mass of the $J / \psi$, the corrections are moderate in size $[30,31]$. While the next-to-leading order corrections are large and have strong dependencies in $p_{T}$ when $p_{T}$ is much larger than $m_{c}$, these large corrections are well understood in terms of the factorized expansion in powers of $1 / p_{T}$ [48]. And, from the factorization theorems of perturbative QCD [44], we do not expect large corrections enhanced by even more powers of $p_{T} / m_{c}$ to appear at higher orders in $\alpha_{s}$. Furthermore, it has been shown that the resummation of leading logarithms of $p_{T} / m_{c}$ does not impact the shape of the short-distance coefficients significantly within the kinematical ranges where data is currently available [39, 49]. Hence, it is doubtful that higher-order corrections in $\alpha_{S}$ can affect the short-distance coefficients significantly at large $p_{T}$. It is still possible that higher order corrections in $\alpha_{s}$ change the relative sizes of contributions at leading power and next-to-leading power in $1 / p_{T}$ and change the shape of the short-distance coefficients, which would affect the determinations of long-distance matrix elements from data. While a complete calculation of next-to-next-to-leading order corrections is currently out of reach, there have been some progress in the calculation of the single-parton fragmentation functions for the ${ }^{1} S_{0}^{[1]}$ and ${ }^{1} S_{0}^{[8]}$ channels to next-to-leading order in $\alpha_{s}[70,71,72]$, which allow us to compute the next-to-nextto-leading order correction to the short-distance coefficients for those channels at leading power in $1 / p_{T}$.

Since in $J / \psi$ hadroproduction, the color-octet channels have larger contributions to the cross section than the color-singlet channel, one may also doubt the convergence of the expansion in powers of the velocity $v$. Including contributions of higher orders in $v$ in the factorization formula (1.1) would result in a huge increase of nonperturbative unknowns, and consequently, a loss of predictive power. It should be noted, however, that none of the determinations of the $J / \psi$ longdistance matrix elements that we considered violate the velocity-power counting, as in all cases, the color-octet long-distance matrix elements are at least an order of magnitude smaller than the color-singlet matrix element, which is consistent with $v^{4} \approx 0.1$. The enhancement of the color-octet channel contributions is of dynamical origin, and is process dependent; in $J / \psi$ photoproduction or $\eta_{c}$ hadroproduction, we do not see such a dramatic suppression of the color-singlet channel contribution. An alternate formulation devised to improve the convergence of the velocity expansion has been suggested in Ref. [73].

In Refs. [74, 75, 76], the authors employed the color glass condensate framework [77, 78, 79, 80] to describe $J / \psi$ hadroproduction for $p_{T}$ comparable to, or even smaller than, the $J / \psi$ mass, where the short-distance process is sensitive to the proton structure at very small Bjorken $x$. Based on the long-distance matrix elements determined in Ref. [32], where all color-octet long-distance matrix elements are positive, the authors found good agreement with LHC data for both cross section and polarization at small $p_{T}$. This is in contrast with Ref. [35], where the standard collinear factorization was used to describe hadron collisions with parton distribution functions; in Ref. [35], the authors found that the color-octet long-distance matrix element must have different signs in order to describe low- $p_{T}$ cross section data. This may hint that for $J / \psi$ hadroproduction at small $p_{T}$, collinear factorization might be unsuitable for describing hadron collisions. It is however yet un- 
known whether there would be such large corrections to photoproduction at HERA, or production processes in lepton colliders.

So far, studies of the color-octet long-distance matrix elements were limited to phenomenological determinations. It is therefore highly desirable to have a constraint, or even a calculation, of the long-distance matrix elements from first principles. Recent developments such as the possible lattice calculation of parton distribution functions [81, 82, 83, 84] give hope that a first-principles determination of nonperturbative NRQCD long-distance matrix elements might be achievable through a thorough investigation of nonrelativistic effective field theories and lattice QCD. For example, the potential NRQCD effective field theory [85], through the separation of scales $m v$ and $m v^{2}$, can reveal previously unknown properties and symmetries of the NRQCD longdistance matrix elements, and may give rise to formulations of the long-distance matrix elements that can be computed in lattice QCD.

\section{Acknowledgments}

I would like to thank Nora Brambilla for her valuable comments and careful reading of the manuscript. This work was supported by the Alexander von Humboldt Foundation and the DFG cluster of excellence 'Origin and Structure of the Universe' (www.universe-cluster.de).

\section{References}

[1] N. Brambilla et al. [Quarkonium Working Group], hep-ph/0412158.

[2] N. Brambilla et al., Eur. Phys. J. C 71, 1534 (2011) [arXiv:1010.5827 [hep-ph]].

[3] T. Matsui and H. Satz, Phys. Lett. B 178, 416 (1986).

[4] M. Drees and M. M. Nojiri, Phys. Rev. D 49, 4595 (1994) [hep-ph/9312213].

[5] W. E. Caswell and G. P. Lepage, Phys. Lett. 167B, 437 (1986).

[6] G. T. Bodwin, E. Braaten and G. P. Lepage, Phys. Rev. D 51, 1125 (1995) Erratum: [Phys. Rev. D 55, 5853 (1997)] [hep-ph/9407339].

[7] P. Pakhlov et al. [Belle Collaboration], Phys. Rev. D 79, 071101 (2009) [arXiv:0901.2775 [hep-ex]].

[8] J. Abdallah et al. [DELPHI Collaboration], Phys. Lett. B 565, 76 (2003) [hep-ex/0307049].

[9] S. Chekanov et al. [ZEUS Collaboration], Eur. Phys. J. C 27 (2003) 173 [hep-ex/0211011].

[10] C. Adloff et al. [H1 Collaboration], Eur. Phys. J. C 25, 25 (2002) [hep-ex/0205064].

[11] F. D. Aaron et al. [H1 Collaboration], Eur. Phys. J. C 68, 401 (2010) [arXiv:1002.0234 [hep-ex]].

[12] F. Abe et al. [CDF Collaboration], Phys. Rev. Lett. 79, 572 (1997).

[13] D. Acosta et al. [CDF Collaboration], Phys. Rev. D 71, 032001 (2005) [hep-ex/0412071].

[14] A. Adare et al. [PHENIX Collaboration], Phys. Rev. D 82, 012001 (2010) [arXiv:0912.2082 [hep-ex]].

[15] B. I. Abelev et al. [STAR Collaboration], Phys. Rev. C 80, 041902 (2009) [arXiv:0904.0439 [nucl-ex]]. 
[16] V. Khachatryan et al. [CMS Collaboration], Eur. Phys. J. C 71, 1575 (2011) [arXiv:1011.4193 [hep-ex]].

[17] S. Chatrchyan et al. [CMS Collaboration], JHEP 1202, 011 (2012) [arXiv:1111.1557 [hep-ex]].

[18] V. Khachatryan et al. [CMS Collaboration], Phys. Rev. Lett. 114, no. 19, 191802 (2015) [arXiv:1502.04155 [hep-ex]].

[19] A. M. Sirunyan et al. [CMS Collaboration], Phys. Lett. B 780, 251 (2018) [arXiv:1710.11002 [hep-ex]].

[20] G. Aad et al. [ATLAS Collaboration], Nucl. Phys. B 850, 387 (2011) [arXiv:1104.3038 [hep-ex]].

[21] G. Aad et al. [ATLAS Collaboration], Eur. Phys. J. C 76, no. 5, 283 (2016) [arXiv:1512.03657 [hep-ex]].

[22] E. Scomparin [ALICE Collaboration], Nucl. Phys. Proc. Suppl. 214, 56 (2011).

[23] K. Aamodt et al. [ALICE Collaboration], Phys. Lett. B 704, 442 (2011) Erratum: [Phys. Lett. B 718, 692 (2012)] [arXiv:1105.0380 [hep-ex]]. 10.1016/j.physletb.2012.10.060;

[24] B. Abelev et al. [ALICE Collaboration], Phys. Lett. B 718, 295 (2012) Erratum: [Phys. Lett. B 748, 472 (2015)] [arXiv:1203.3641 [hep-ex]]. 10.1016/j.physletb.2015.06.058;

[25] B. Abelev et al. [ALICE Collaboration], JHEP 1211, 065 (2012) [arXiv:1205.5880 [hep-ex]].

[26] R. Aaij et al. [LHCb Collaboration], Eur. Phys. J. C 71, 1645 (2011) [arXiv:1103.0423 [hep-ex]].

[27] R. Aaij et al. [LHCb Collaboration], JHEP 1302, 041 (2013) [arXiv:1212.1045 [hep-ex]].

[28] R. Aaij et al. [LHCb Collaboration], JHEP 1306, 064 (2013) [arXiv:1304.6977 [hep-ex]].

[29] Y.-Q. Ma, K. Wang, and K.-T. Chao, Phys. Rev. D 84, 114001 (2011) [arXiv:1012.1030 [hep-ph]].

[30] M. Butenschoen and B. A. Kniehl, Phys. Rev. Lett. 106, 022003 (2011) [arXiv:1009.5662 [hep-ph]].

[31] Y.-Q. Ma, K. Wang, and K.-T. Chao, Phys. Rev. Lett. 106, 042002 (2011) [arXiv:1009.3655 [hep-ph]].

[32] K.-T. Chao, Y.-Q. Ma, H.-S. Shao, K. Wang, and Y.-J. Zhang, Phys. Rev. Lett. 108, 242004 (2012) [arXiv:1201.2675 [hep-ph]].

[33] M. Butenschoen and B. A. Kniehl, Phys. Rev. Lett. 108, 172002 (2012) [arXiv:1201.1872 [hep-ph]].

[34] B. Gong, L.-P. Wan, J.-X. Wang, and H.-F. Zhang, Phys. Rev. Lett. 110, 042002 (2013) [arXiv:1205.6682 [hep-ph]].

[35] M. Butenschoen and B. A. Kniehl, Phys. Rev. D 84, 051501 (2011) [arXiv:1105.0820 [hep-ph]].

[36] M. Butenschoen and B. A. Kniehl, Phys. Rev. Lett. 104, 072001 (2010) [arXiv:0909.2798 [hep-ph]].

[37] M. Butenschoen and B. A. Kniehl, Phys. Rev. Lett. 107, 232001 (2011) [arXiv:1109.1476 [hep-ph]].

[38] H. S. Shao, H. Han, Y. Q. Ma, C. Meng, Y. J. Zhang and K. T. Chao, JHEP 1505, 103 (2015) [arXiv:1411.3300 [hep-ph]].

[39] G. T. Bodwin, K. T. Chao, H. S. Chung, U. R. Kim, J. Lee and Y. Q. Ma, Phys. Rev. D 93, no. 3, 034041 (2016) [arXiv:1509.07904 [hep-ph]].

[40] G. T. Bodwin, H. S. Chung, D. Kang, J. Lee and C. Yu, Phys. Rev. D 77, 094017 (2008) [arXiv:0710.0994 [hep-ph]].

[41] E. J. Eichten and C. Quigg, Phys. Rev. D 52, 1726 (1995) [hep-ph/9503356]. 
[42] M. Butenschoen and B. A. Kniehl, Mod. Phys. Lett. A 28, 1350027 (2013) [arXiv:1212.2037 [hep-ph]].

[43] G. T. Bodwin, H. S. Chung, U. R. Kim and J. Lee, Phys. Rev. D 92, no. 7, 074042 (2015) [arXiv:1504.06019 [hep-ph]].

[44] J. C. Collins and D. E. Soper, Nucl. Phys. B 194, 445 (1982).

[45] Z. B. Kang, J. W. Qiu and G. Sterman, Phys. Rev. Lett. 108, 102002 (2012) [arXiv:1109.1520 [hep-ph]].

[46] S. Fleming, A. K. Leibovich, T. Mehen and I. Z. Rothstein, Phys. Rev. D 86, 094012 (2012) [arXiv:1207.2578 [hep-ph]].

[47] Z. B. Kang, Y. Q. Ma, J. W. Qiu and G. Sterman, Phys. Rev. D 90, no. 3, 034006 (2014) [arXiv:1401.0923 [hep-ph]].

[48] Y. Q. Ma, J. W. Qiu, G. Sterman and H. Zhang, Phys. Rev. Lett. 113, no. 14, 142002 (2014) [arXiv:1407.0383 [hep-ph]].

[49] G. T. Bodwin, H. S. Chung, U. R. Kim and J. Lee, Phys. Rev. Lett. 113, no. 2, 022001 (2014) [arXiv:1403.3612 [hep-ph]].

[50] E. Braaten and S. Fleming, Phys. Rev. Lett. 74, 3327 (1995) [hep-ph/9411365].

[51] T. Affolder et al. [CDF Collaboration], Phys. Rev. Lett. 85, 2886 (2000) [hep-ex/0004027].

[52] A. Abulencia et al. [CDF Collaboration], Phys. Rev. Lett. 99, 132001 (2007) [arXiv:0704.0638 [hep-ex]].

[53] B. Abelev et al. [ALICE Collaboration], Phys. Rev. Lett. 108, 082001 (2012) [arXiv:1111.1630 [hep-ex]].

[54] S. Acharya et al. [ALICE Collaboration], Eur. Phys. J. C 78, no. 7, 562 (2018) [arXiv:1805.04374 [hep-ex]].

[55] S. Chatrchyan et al. [CMS Collaboration], Phys. Lett. B 727, 381 (2013) [arXiv:1307.6070 [hep-ex]].

[56] R. Aaij et al. [LHCb Collaboration], Eur. Phys. J. C 73, no. 11, 2631 (2013) [arXiv:1307.6379 [hep-ex]].

[57] R. Aaij et al. [LHCb Collaboration], JHEP 1712, 110 (2017) [arXiv:1709.01301 [hep-ex]].

[58] M. Butenschoen and B. A. Kniehl, Nucl. Phys. Proc. Suppl. 222-224, 151 (2012) [arXiv:1201.3862 [hep-ph]].

[59] M. Baumgart, A. K. Leibovich, T. Mehen and I. Z. Rothstein, JHEP 1411, 003 (2014) [arXiv:1406.2295 [hep-ph]].

[60] R. Aaij et al. [LHCb Collaboration], Phys. Rev. Lett. 118, no. 19, 192001 (2017) [arXiv:1701.05116 [hep-ex]].

[61] CMS Collaboration [CMS Collaboration], CMS-PAS-HIN-18-012.

[62] R. Bain, L. Dai, A. Leibovich, Y. Makris and T. Mehen, Phys. Rev. Lett. 119, no. 3, 032002 (2017) [arXiv:1702.05525 [hep-ph]].

[63] M. Procura and I. W. Stewart, Phys. Rev. D 81, 074009 (2010) Erratum: [Phys. Rev. D 83, 039902 (2011)] [arXiv:0911.4980 [hep-ph]]. 
[64] R. Bain, L. Dai, A. Hornig, A. K. Leibovich, Y. Makris and T. Mehen, JHEP 1606, 121 (2016) [arXiv:1603.06981 [hep-ph]].

[65] R. Aaij et al. [LHCb Collaboration], Eur. Phys. J. C 75, no. 7, 311 (2015) [arXiv:1409.3612 [hep-ex]].

[66] M. Butenschoen, Z. G. He and B. A. Kniehl, Phys. Rev. Lett. 114, no. 9, 092004 (2015) [arXiv:1411.5287 [hep-ph]].

[67] H. Han, Y. Q. Ma, C. Meng, H. S. Shao and K. T. Chao, Phys. Rev. Lett. 114, no. 9, 092005 (2015) [arXiv:1411.7350 [hep-ph]].

[68] H. F. Zhang, Z. Sun, W. L. Sang and R. Li, Phys. Rev. Lett. 114, no. 9, 092006 (2015) [arXiv:1412.0508 [hep-ph]].

[69] Y. Feng and H. F. Zhang, arXiv:1809.04894 [hep-ph].

[70] P. Artoisenet and E. Braaten, JHEP 1504, 121 (2015) [arXiv:1412.3834 [hep-ph]].

[71] P. Artoisenet and E. Braaten, arXiv:1810.02448 [hep-ph].

[72] P. Zhang, C. Y. Wang, X. Liu, Y. Q. Ma, C. Meng and K. T. Chao, arXiv:1810.07656 [hep-ph].

[73] Y. Q. Ma and K. T. Chao, arXiv:1703.08402 [hep-ph].

[74] Z. B. Kang, Y. Q. Ma and R. Venugopalan, JHEP 1401, 056 (2014) [arXiv:1309.7337 [hep-ph]].

[75] Y. Q. Ma and R. Venugopalan, Phys. Rev. Lett. 113, no. 19, 192301 (2014) [arXiv:1408.4075 [hep-ph]].

[76] Y. Q. Ma, T. Stebel and R. Venugopalan, arXiv:1809.03573 [hep-ph].

[77] Y. V. Kovchegov and E. Levin, Camb. Monogr. Part. Phys. Nucl. Phys. Cosmol. 33 (2012).

[78] F. Gelis, E. Iancu, J. Jalilian-Marian and R. Venugopalan, Ann. Rev. Nucl. Part. Sci. 60, 463 (2010) [arXiv:1002.0333 [hep-ph]].

[79] H. Weigert, Prog. Part. Nucl. Phys. 55, 461 (2005) [hep-ph/0501087].

[80] E. Iancu and R. Venugopalan, In *Hwa, R.C. (ed.) et al.: Quark gluon plasma* 249-3363 [hep-ph/0303204].

[81] X. Ji, Phys. Rev. Lett. 110, 262002 (2013) [arXiv:1305.1539 [hep-ph]].

[82] A. Radyushkin, Phys. Lett. B 767, 314 (2017) [arXiv:1612.05170 [hep-ph]].

[83] A. J. Chambers et al., Phys. Rev. Lett. 118, no. 24, 242001 (2017) [arXiv:1703.01153 [hep-lat]].

[84] Y. Q. Ma and J. W. Qiu, Phys. Rev. Lett. 120, no. 2, 022003 (2018) [arXiv:1709.03018 [hep-ph]].

[85] N. Brambilla, A. Pineda, J. Soto and A. Vairo, Nucl. Phys. B 566, 275 (2000) [hep-ph/9907240]. 